\title{
Let-7g-5p inhibits epithelial-mesenchymal transition consistent with reduction of glioma stem cell phenotypes by targeting VSIG4 in glioblastoma
}

\author{
XIN-HUA ZHANG ${ }^{1}$, YUN QIAN ${ }^{1}$, ZHENG LI ${ }^{1}$, NING-NING ZHANG ${ }^{1}$ and YUN-JIE XIE ${ }^{2}$ \\ ${ }^{1}$ Department of Neurosurgery, The Affiliated Hospital of Taishan Medical University, Tai'an, Shandong 271000; \\ ${ }^{2}$ Department of Neurosurgery, Jining No. 1 People's Hospital, Jining, Shandong 272011, P.R. China
}

Received April 27, 2016; Accepted September 2, 2016

DOI: $10.3892 /$ or.2016.5098

\begin{abstract}
Epithelial-mesenchymal transition (EMT) and stem-like glioma cells display hallmark therapeutic resistance. Understanding of the mechanisms underlying these properties will be vital for the development of effective therapies. In this study, we found that VSIG4 protein is upregulated in glioblastoma. Overexpressing VSIG4 induced EMT and significantly promoted invasion and migration in glioblastoma U-87MG cells. Moreover, we showed that its overexpression promoted formation of glioma stem cell phenotypes in U-87MG cells. P4HB, VAMP8 and Connexin 43 (CX43) can promote temozolomide (TMZ) resistance in human glioma cells. We showed that P4HB, VAMP8 and CX43 protein were upregulated by VSIG4 in U-87MG cells, implying its upregulation might be a cause for temozolomide resistance. We found that let-7g-5p can inhibit VSIG4 protein expression, but it cannot degrade VSIG4 mRNA in U-87MG cells. Contrary to VSIG4, we demonstrated that overexpressing let-7g-5p promoted mesenchymal-epithelial transition (MET) and significantly inhibited invasion and migration consistent with the reduction of glioblastoma stem cell phenotypes in U-87MG cells. Thus, we concluded that let-7g-5p inhibits epithelial-mesenchymal transition (EMT) consistent with reduction of glioma stem cell (GSC) phenotypes by targeting VSIG4 in glioblastoma.
\end{abstract}

\section{Introduction}

Glioblastoma multiforme (GBM) is the most common form of human primary malignant brain tumors and it accounts for $>60 \%$ of all primary brain tumors in adults $(1,2)$. Because of resistance to conventional therapies, the prognosis of GBM

Correspondence to: Dr Yun-Jie Xie, Department of Neurosurgery, Jining No. 1 People's Hospital, 6 Jiankang Road, Jining, Shandong 272011, P.R. China

E-mail: xieyunjiejn@163.com

Key words: epithelial-mesenchymal transition, glioma stem cells, VSIG4, let-7g-5p, glioblastoma remains dismal with median survival of $\sim 14$ months and 5-year survival only $\sim 3 \%$ (3). Understanding molecular mechanisms underpinning resistance of conventional therapies of glioblastoma will offer novel targets for effective therapies.

MicroRNAs (miRNAs) are small, non-coding RNAs that post-transcriptionally regulate gene expression (4) and play significant roles in maintaining normal cellular functions (5). Deregulation of miRNA expression leads to diverse disease types, including cancers (6) as exemplified by their differential expression in carcinomas $(7)$, sarcomas $(8,9)$, and hematologic tumors (10). Let-7g-5p is significantly downregulated in the serum of GBM patients and it has been proposed as a tumor suppressive gene $(11,12)$.

Glioma stem cells (GSCs) or glioma initiating cells (GICs) have been identified and shown to constitute a primitive cell population capable of self-renewal and differentiation that has the unique capacity to give rise to new tumors upon serial transplantation (13-16). Cancer stem/initiating cells are believed to play an essential role in tumor recurrence after therapeutic intervention (17), and their high chemo-resistance and radiation resistance (18) require the identification of alternative therapeutic strategies that could effectively lead to their functional or physical eradication. Although a few signaling pathways, including Sonic-Hedgehog (19), and the bone morphogenic proteins BMP4 and BMPR1B $(20,21)$ have been shown to be implicated in GSCs maintenance, the mechanisms underlying GSCs generation, and propagation have yet to be elucidated.

Epithelial to mesenchymal transition (EMT) is an essential process for driving plasticity during development, but is also an unintentional behavior of cells during progression of malignant tumor (22-24). EMT confers mesenchymal properties on epithelial cells and has been closely associated with the acquisition of aggressive traits by carcinoma cells (25). Disturbance of a controlled epithelial balance is triggered by altering several layers of regulation, including the transcriptional and translational machinery, expression of non-coding RNAs, alternative splicing and protein stability (26-28).

In this study, we found that VSIG4 protein is upregulated in glioblastoma. Overexpressing VSIG4 induced epithelialmesenchymal transition (EMT) and significantly promoted invasion and migration in glioblastoma U-87MG cells. 
Moreover, we showed that its overexpression promoted formation of glioma stem cell phenotypes in U-87MG cells. We found that let-7g-5p can downregulate VSIG4 protein expression, but it cannot degrade VSIG4 mRNA in U-87MG cells. Contrary to VSIG4, we demonstrated that overexpressing let-7g-5p promoted mesenchymal-epithelial transition (MET) and significantly inhibited invasion and migration consistent with the reduction of glioblastoma stem cell phenotypes in U-87MG cells.

\section{Materials and methods}

Glioblastoma tissues. Glioblastoma tissues and adjacent normal tissues were obtained from the Department of Neurosurgery, The Affiliated Hospital of Taishan Medical University, Shandong, China. All tissues were examined histologically, and pathologists confirmed the diagnosis. Medical ethics committee approved the experiments. The use of human's tissue samples follows internationally recognized guidelines as well as local and national regulations. Informed consent was obtained from each individual.

Glioblastoma U-87MG cell line, VSIG4 expressing plasmids/ empty vectors, pre-let- $7 \mathrm{~g}-5 \mathrm{p} / \mathrm{control} \mathrm{miR}$ and transfection. Human glioblastoma cell line U-87MG was obtained from American Type Culture Collection. Briefly, cells were maintained in RPMI-1640 medium supplemented with $10 \%$ fetal bovine serum (FBS) (Gibco, Grand Island, NY, USA) and penicillin/streptomycin at $37^{\circ} \mathrm{C}$ in a humidified atmosphere with $5 \% \mathrm{CO}_{2}$. VSIG4 expressing plasmids/empty vectors (pcDNA3.1) were purchased from Tiangen (Beijing, China). Pre-let-7g and control miR were purchased from Ambion, Inc. (Ambion, Austin, TX, USA). For transfection experiments, the cells were cultured in serum-free medium without antibiotics at $60 \%$ confluence for $24 \mathrm{~h}$, and then transfected with transfection reagent (Lipofectamine 2000, Invitrogen, Carlsbad, CA, USA) according to the manufacturer's instructions. After incubation for $6 \mathrm{~h}$, the medium was removed and replaced with normal culture medium for $48 \mathrm{~h}$, unless otherwise specified.

Western blot analysis. Western blot analysis was performed as described before (29). Briefly, after incubation with primary antibody anti-VSIG4 (1:500; Abcam, Cambridge, MA, USA), anti-CD133 (1:500; Abcam), anti-EZH2 (1:500; Abcam), anti-cMet (1:500; Abcam), anti-P4HB (1:500; Abcam), anti-VAMP8 (1:500; Abcam), anti-CX43 (1:500; Abcam), anti-E-cadherin (1:500; Abcam), anti-TGFB1 (1:500; Abcam), anti-vimentin (1:500; Abcam), anti-SNAIL (1:500; Abcam), anti-Notch1 (1:500; Abcam), anti-TLR9 (1:500; Abcam), anti-EphA2 (1:500; Abcam), anti-MLK4 (1:500; Abcam) and anti- $\beta$-actin (1:500; Abcam) overnight at $4^{\circ} \mathrm{C}$, IRDye ${ }^{\mathrm{TM}}-800$ conjugated anti-rabbit secondary antibodies (LI-COR, Biosciences, Lincoln, NE, USA) were used for $30 \mathrm{~min}$ at room temperature. The specific proteins were visualized by Odyssey ${ }^{\mathrm{TM}}$ Infrared Imaging System (Gene Co., Lincoln, NE, USA).

Sphere growth. Cells $\left(10^{3} / \mathrm{ml}\right)$ in serum-free RPMI-1640/1 mM Na-pyruvate were seeded on $0.5 \%$ agar precoated 6 -well plates. After 1 week, half the medium was changed every third day. Single spheres were picked and counted.
Immunofluorescence analyses. For U-87MG cell immunofluorescence analyses, U-87MG cells were plated on glass coverslips in 6-well plates and transfected as indicated. At $48 \mathrm{~h}$ after transfection, coverslips were stained with CD44 (1:500; Abcam) or antibody anti-VSIG4 (1:500; Abcam). Alexa Fluor 488 goat anti-rabbit IgG antibody was used as secondary antibody (Invitrogen). Coverslips were counterstained with DAPI (Invitrogen-Molecular Probes, Eugene, OR, USA) for visualization of the nuclei. Microscopic analysis was performed with a confocal laser-scanning microscope (Leica Microsystems, Bensheim, Germany). Fluorescence intensities were measured in a few viewing areas for 300 cells per coverslip and analyzed using ImageJ $1.37 \mathrm{v}$ software (http://rsb.info. nih.gov/ij/index.html).

Wound healing assay. Wound healing assay was performed as described before (30).

Migration and invasion assay. Migration and invasion assay was performed as described before (29).

Methods of bioinformatics. The analysis of potential microRNA target site using the commonly used prediction algorithms - miRanda (http://www.microrna.org/).

Real-time PCR for microRNAs. Total RNA from cultured cells, with efficient recovery of small RNAs, was isolated using the mirVana miRNA Isolation kit (Ambion). Detection of the mature form of miRNAs was performed using the mirVana qRT-PCR miRNA Detection kit and qRT-PCR Primer Sets, according to the manufacturer's instructions (Ambion). The U6 small nuclear RNA was used as an internal control.

Reverse transcription-polymerase chain reaction. It was performed as described before (31). Primers for VSIG4: forward, 5'-GTGTCCAGTTTGGCTAGTGCC-3'; reverse, 5'-GACTGGAGAACAGAAGCAGGC-3'. Primers for GAPDH: forward, 5'-CGGAGTCAACGGATTTGGTCG TAT-3'; reverse, 5'-AGCCTTCTCCATGGTGGTGAAGAC-3'.

Northern blot analysis. Northern blot analysis for miRNAs were performed as described previously (32). Probes were labeled with $\left[\gamma_{-}{ }^{32} \mathrm{P}\right]-\mathrm{ATP}$ complementary to let-7g-5p and U6 snRNA.

Statistical analysis. Data are presented as mean \pm SEM. Student's t-test (two-tailed) was used to compare two groups $(\mathrm{P}<0.05$ was considered significant), unless otherwise indicated $\left(\chi^{2}\right.$ test $)$.

\section{Results}

VSIG4 promotes formation of stem cell-like population in glioblastoma U-87MG cells. In an attempt to identify VSIG4 protein expression between glioblastoma tissues and adjacent normal tissues, we performed western blotting in tumor tissues versus normal tissues. Protein was isolated from 6 pairs of glioblastoma tissues and normal tissues (patient nos. 1-6). We found that VSIG4 protein was significantly increased in cancer 


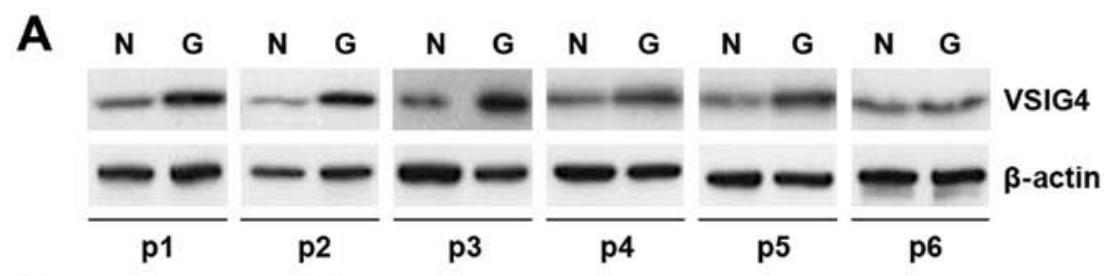

B

C
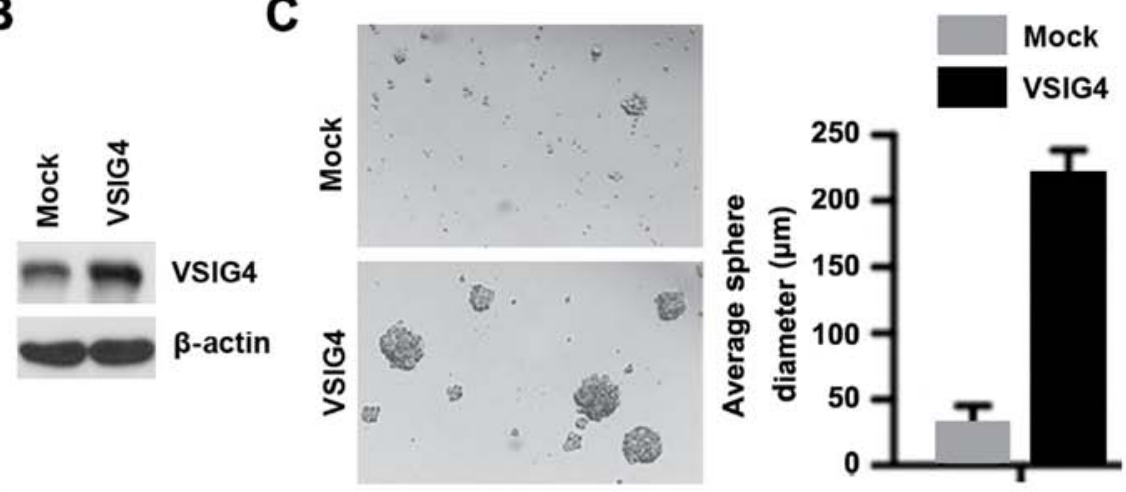

D

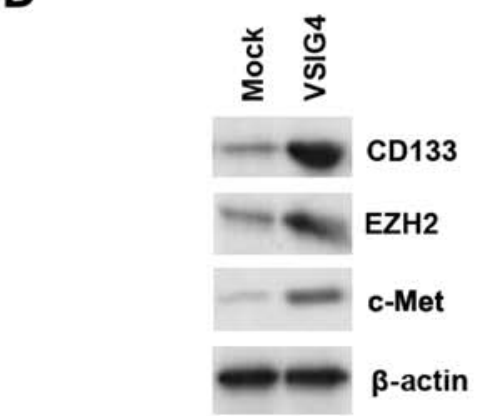

E

$\mathbf{F}$
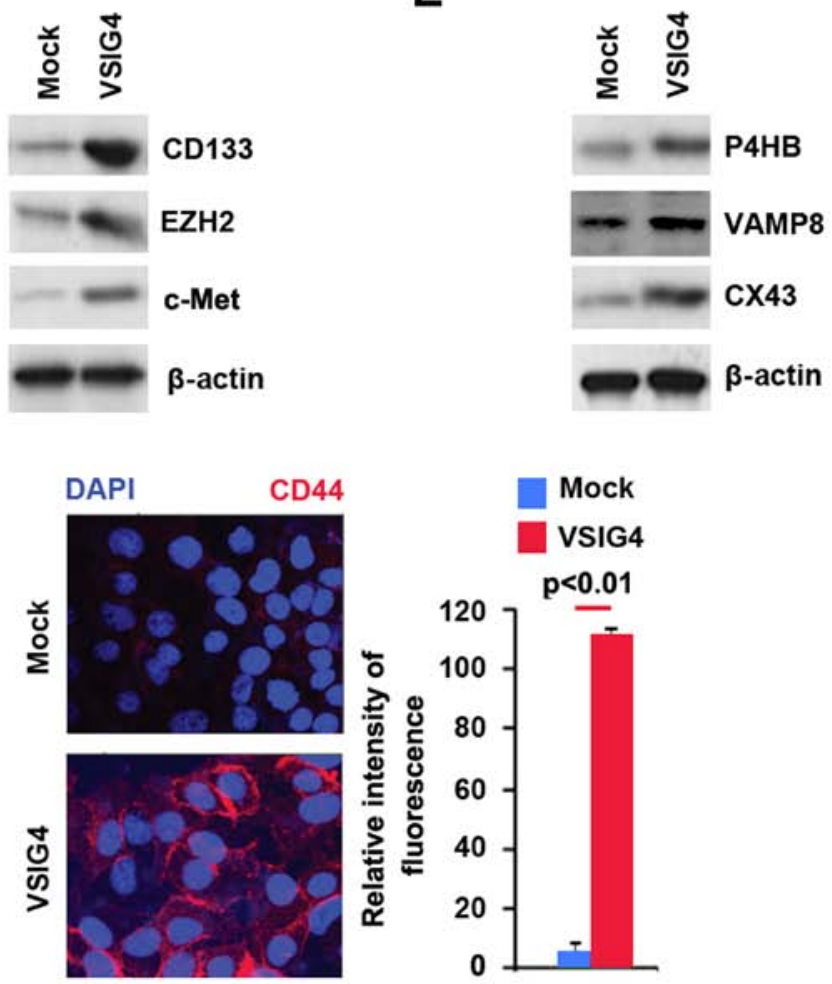

Figure 1. VSIG4 promotes formation of stem cell-like population in glioblastoma U-87MG cells. (A) Western blotting for VSIG4 in glioblastoma tissues (C) and adjacent normal tissues (N). Patients were numbered 1-6. All the 6 patients were diagnosed with glioblastoma. $\beta$-actin was a loading control. $n=6$. (B) Western blotting for VSIG4 in U-87MG cells transfected with VSIG4 expressing plasmids or empty vectors (mock). $\beta$-actin was a loading control. $\mathrm{n}=3$. (C) Sphere growth for U-87MG transfected with VSIG4 expressing plasmids or empty vectors (mock). (D) Western blotting for CD133, EZH2 and c-Met in U-87MG cells transfected with VSIG4 expressing plasmids or empty vectors (mock). $\beta$-actin was a loading control. $n=3$. (E) Western blotting for P4HB, VAMP8 and CX43 in U-87MG cells transfected with VSIG4 expressing plasmids or empty vectors (mock). $\beta$-actin was a loading control. $n=3$. (F) Immunofluorescence analyses for CD44 in U-87MG cells transfected with VSIG4 expressing plasmids or empty vectors (mock). $n=3$.

tissues, compared with adjacent normal tissues (Fig. 1A). It implied that VSIG4 could be an oncogene in glioblastoma.

In order to assess the role of VSIG4 in glioblastoma, we transfected U-87MG cells with VSIG4 expressing plasmids and then western blotting was performed. We found that VSIG4 protein was significantly increased in the cells transfected with VSIG4 expressing plasmids (Fig. 1B). To determine whether
VSIG4 can affect GSCs, we performed sphere forming assay to assess formation of stem cell-like population. We found that formations of spheres were increased by VSIG4 in U-87MG cells (Fig. 1C). CD133, EZH2, c-Met and CD44 are robust markers and are of functional importance for GSC for tumor initiation (33-36). In order to detect whether CD133, EZH2, c-Met and CD44 protein expression can be affected by VSIG4, 
A
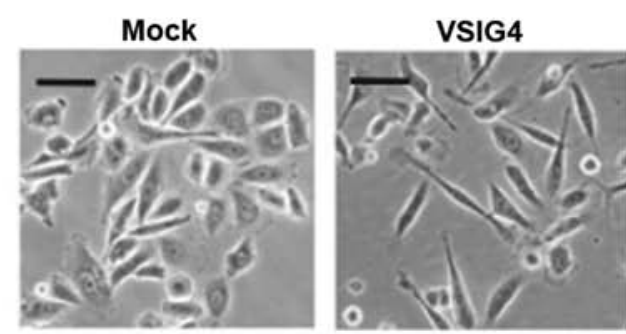

B

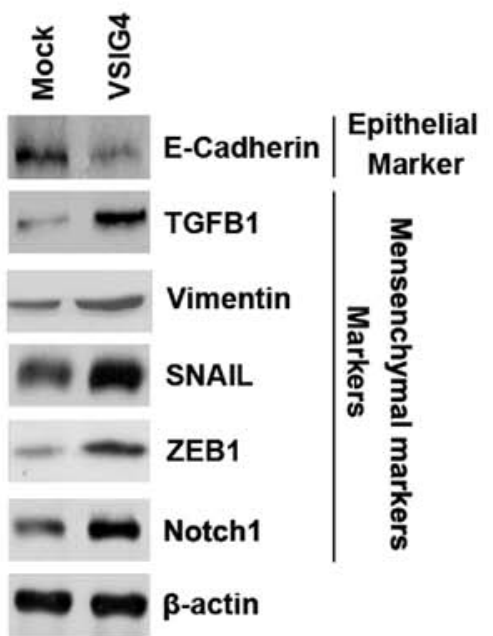

C

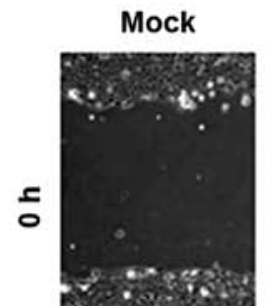

VSIG4
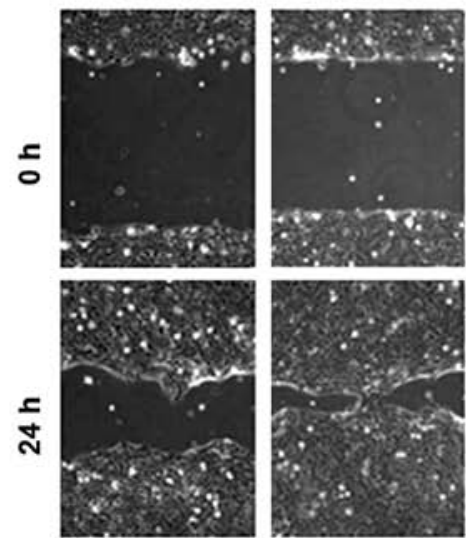

D

\section{E}
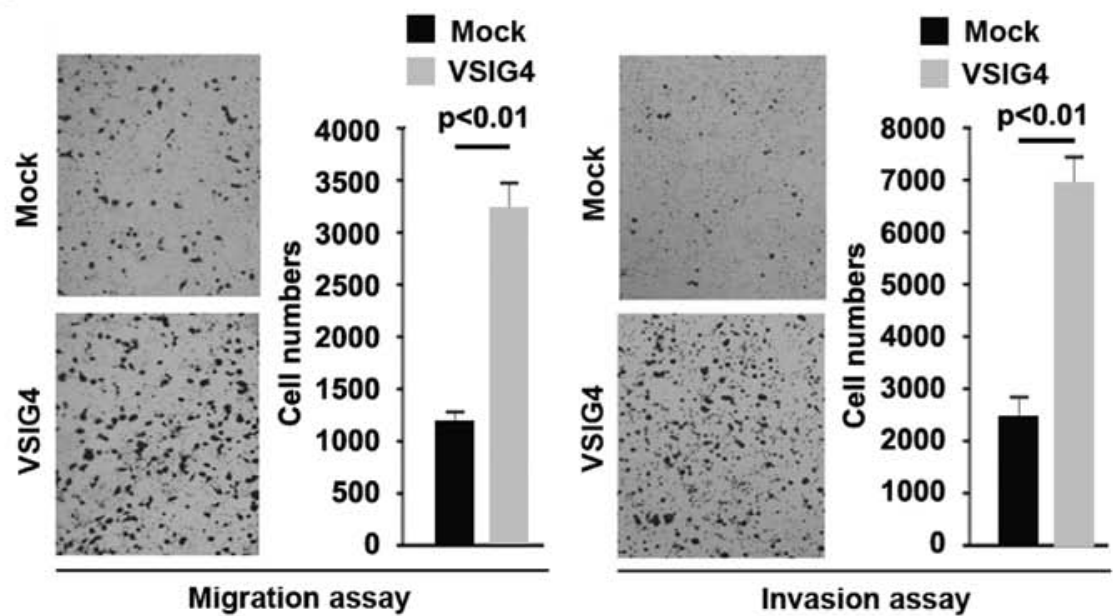

Figure 2. Overexpressing VSIG4 promotes EMT in glioblastoma U-87MG cells. (A) U-87MG cells were transfected with VSIG4 expressing plasmids or empty vectors (mock). Cells were photographed after $72 \mathrm{~h}$ of transfection. $\mathrm{n}=3$. (B) Western blotting for E-cadherin, TGFB1, Vimentin, SNAIL, ZEB1 and Notch1 in U-87MG cells transfected with VSIG4 expressing plasmids or empty vectors (mock). $\beta$-actin was a loading control. $n=3$. (C) Wound-healing assays for U-87MG cells transfected with VSIG4 expressing plasmids or empty vectors (mock). The cell layer was photographed. n=3. (D) Migration assays for U-87MG cells transfected with VSIG4 expressing plasmids or empty vectors (mock). n=3. (E) Invasion assays for U-87MG cells transfected with VSIG4 expressing plasmids or empty vectors (mock). $\mathrm{n}=3$.

we performed western blotting and immunofluorescence. The results showed that CD133, EZH2, c-Met (Fig. 1D) and CD44 protein were upregulated by VSIG4 (Fig. 1F).

P4HB, VAMP8 and Connexin 43 (CX43) can promote temozolomide (TMZ) resistance in human glioma cells (37-39). To identify whether VSIG4 could have potential to affect temozolomide (TMZ) resistance, we performed western blotting to detect P4HB, VAMP8 and Connexin 43 (CX43) protein. The results showed that $\mathrm{P} 4 \mathrm{HB}$, VAMP8 and Connexin 43 (CX43) protein were upregulated by VSIG4 in U-87MG cells (Fig. 1E).
Overexpressing VSIG4 promotes EMT in glioblastoma $U-87 M G$ cells. EMT has been shown to result in cancer cells with stem cell-like characteristics that have a propensity to invade surrounding tissue and display resistance to certain therapeutic interventions (40). In order to assess the role of VSIG4 in EMT of U-87MG, we transfected U-87MG cells with VSIG4 expressing plasmids and then we found that its overexpression caused significant changes in the cell morphology (EMT, phenotype from a cobblestone-like to a spindle-like morphology) (Fig. 2A). To further verify that the changes in 
A

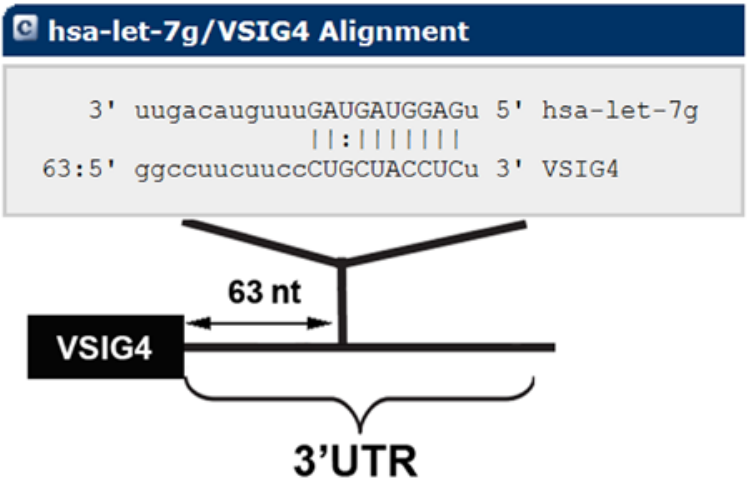

B

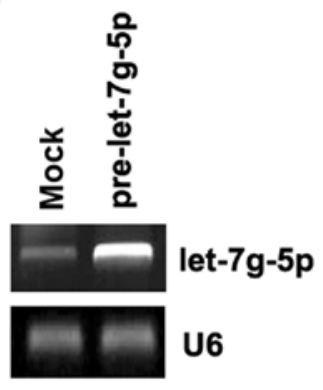

D

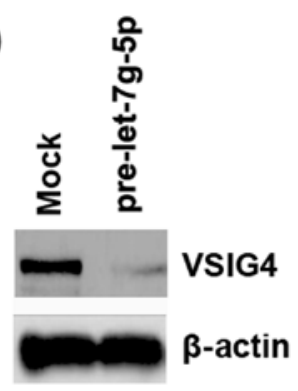

E

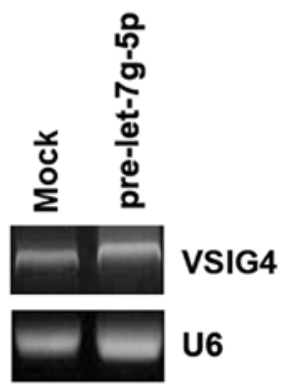

Figure 3. Let-7g-5p inhibits VSIG4 in glioblastoma cells. (A) Schematic of predicted let-7g-5p binding sites in the 3'UTR of VSIG4 mRNA by TargetScan. (B) Real-time RT-PCR for let-7g-5p in U-87MG cells transfected with pre-let-7g-5p or control miR (mock). U6 was a loading control. $\mathrm{n}=3$. (C) Immunofluorescence analyses for VSIG4 in U-87MG cells transfected with pre-let-7g-5p and control miR (mock). Upper panel shows microscopic images of immunofluorescence staining of one representative experiment (x100 magnification). Bottom panel shows graphic presentation of mean fluorescence intensities. $\mathrm{n}=3$. (D) Western blotting for VSIG4 in U-87MG cells transfected with pre-let-7g-5p and control miR (mock). $\beta$-actin was a loading control. $\mathrm{n}=3$. (E) RT-PCR for VSIG4 in U-87MG cells. U-87MG cells were transfected with pre-let-7g-5p and control miR (mock). GAPDH was a loading control. $\mathrm{n}=3$.

cell morphology are caused by EMT, we performed western blotting to detect expression of epithelial and mesenchymal markers in U-87MG cells transfected with VSIG4 expressing plasmids and the cells transfected with empty vectors. The results revealed that epithelial marker (E-cadherin) was inhibited and the mesenchymal markers (TGFB1, Vimentin, SNAIL, ZEB1 and Notch1) were induced by VSIG4 in U-87 MG cells (Fig. 1B).

EMT can result in increased cell invasion and migration (41-43). Thus, we reasoned that VSIG4 could also affect invasion and migration in U-87 MG cells. To identify this reason, we performed would healing, invasion, and migration assays. We found that overexpressing VSIG4 resulted in enhanced migration (Fig. 1C and D) and invasion (Fig. 1E) in the cells.

Let-7g-5p inhibits VSIG4 in glioblastoma U-87MG cells. Having demonstrated that overexpressing VSIG4 promoted formation of stem cell-like population and EMT, next we studied the mechanisms regulating VSIG4 expression in the disease. MicroRNAs (miRs) are a class of small non-coding RNAs ( 22 nucleotides) and negatively regulate protein-coding gene expression by targeting mRNA degradation or translation inhibition (44-46). To further confirm whether VSIG4 could be regulated by microRNA, we used the commonly used prediction algorithm - miRanda (http://www.microrna.org/ microrna/home.do) to analyze 3'UTR of VSIG4. A dozen of microRNAs were found by the algorithm. Nonetheless, we are interested in let- $7 \mathrm{~g}-5 \mathrm{p}$, because let- $7 \mathrm{~g}-5 \mathrm{p}$ has been proposed as a tumor suppressive gene $(47,48)$. However, its role still keeps emerging in glioblastoma.

Target sites on 3'UTR of VSIG4 are shown in Fig. 3A. We reasoned that let-7g-5p could downregulate VSIG4 expression by targeting its 3'UTR in glioblastoma. Downregulation of let-7g-5p can contribute to upregulation of VSIG4 in glioblastoma. In an attempt to identify the role of let- $7 \mathrm{~g}-5 \mathrm{p}$ in regulating VSIG4 expression in glioblastoma, we transfected U-87MG 

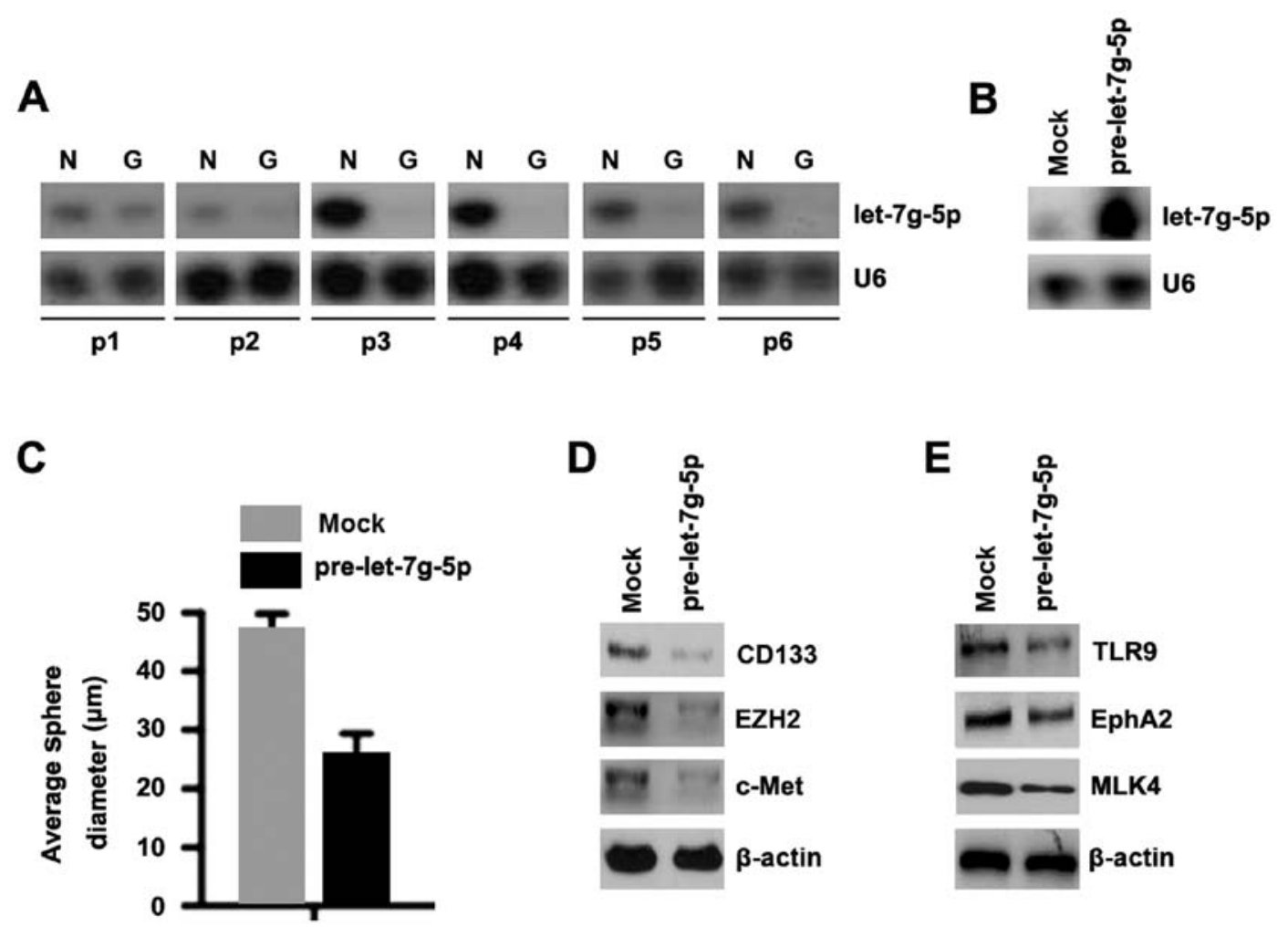

Figure 4. Let-7g-5p inhibits formation of stem cell-like population in glioblastoma U-87MG cells. (A) Northern blotting for let-7g-5p in glioblastoma tissues (C) and adjacent normal tissues $(\mathrm{N})$. Patients were numbered 1-6. All the 6 patients were diagnosed as glioblastoma. U6 was a loading control. $n=6$. (B) Northern blotting for let-7g-5p in U-87MG cells transfected with pre-let-7g-5p and control miR (mock). U6 was a loading control. $\mathrm{n}=3$. (C) Sphere growth for U-87MG cells transfected with pre-let-7g-5p or control miR. (D) Western blotting for CD133, EZH2 and c-Met in U-87MG cells transfected with pre-let-7g-5p and control miR (mock). $\beta$-actin was a loading control. $\mathrm{n}=3$. (E) Western blotting for TLR9, EphA2 and MLK4 in U-87MG cells transfected with pre-let-7g-5p and control miR (mock). $\beta$-actin was a loading control. $n=3$.

cells with pre-let-7g-5p and control miR. After transfection, let-7g-5p expression was detected by real-time PCR and the results showed that let-7g-5p was significantly increased by pre-let-7g-5p in the cells (Fig. 3B).

To confirm the reason, we performed immunofluorescence analyses in U-87MG cells transfected with pre-let-7g-5p and control miR. The results showed that VSIG4 protein was evidently inhibited in the cells transfected with pre-let-7g-5p (Fig. 3C). We next performed western blotting and RT-PCR to detect VSIG4 expression in U-87MG cells transfected with pre-let-7g-5p and control miR. The results showed that VSIG4 protein (Fig. 3D) was significantly downregulated in the cells transfected with pre-let-7g-5p. However, we found that let-7g-5p did not degrade VSIG4 mRNA (Fig. 3E).

Let-7g-5p inhibits formation of stem cell-like population in glioblastoma $U-87 M G$ cells. In an attempt to identify let-7g-5p expression between glioblastoma tissues and adjacent normal tissues, we performed northern blotting in tumor tissues versus normal tissues. Protein was isolated from 6 pairs of glioblastoma tissues and normal tissues (patient nos. 1-6). We found that let-7g-5p was significantly decreased in glioblastoma tissues, compared with adjacent normal tissues (Fig. 4A). It indicated that let- $7 \mathrm{~g}-5 \mathrm{p}$ could be a tumor suppressive gene in glioblastoma. In order to assess the role of let- $7 \mathrm{~g}-5 \mathrm{p}$ in glioblastoma, we transfected U-87MG cells with pre-let-7g-5p and then northern blot analyses were performed. We found that let-7g-5p was significantly increased in the cells transfected with pre-let-7g-5p (Fig. 4B).

To determine whether let-7g-5p could affect stem-like cell characteristics, we performed sphere forming assay to assess the capacity of CSC or CSC-like cell self-renewal in this study. We found that formations of spheres were decreased by let-7g-5p in U-87MG cells (Fig. 4C). We also performed western blotting to detect whether GSCs markers, CD133, EZH2, c-MET, TLR9, EphA2 and MLK4 can be affected by let-7g-5p in the cells. The results showed that CD133, EZH2, c-MET, TLR9, EphA2 and MLK4 protein was significantly decreased by let-7g-5p in U-87MG cells (Fig. 5D and E).

Overexpressing let-7g-5p promotes MET in glioblastoma $U-87 M G$ cells. To assess the role of let-7g-5p in U-87MG cells, we transfected U-87MG cells with pre-let-7g-5p and control miR. We found that its overexpression caused slight changes in the cell morphology (MET, phenotype from a spindle-like morphology to a cobblestone-like) (Fig. 5A). To further verify that the changes in cell morphology are caused by MET, we performed western blotting to detect expression levels of epithelial and mesenchymal markers in U-87MG cells transfected with pre-let-7g-5p and the cells transfected with control miR. The results revealed that epithelial marker (E-cadherin) was induced and the mesenchymal markers (TGFB1, Vimentin, SNAIL, ZEB1 and Notch1) were inhibited by let-7g-5p in U-87 MG cells (Fig. 5B). 
A
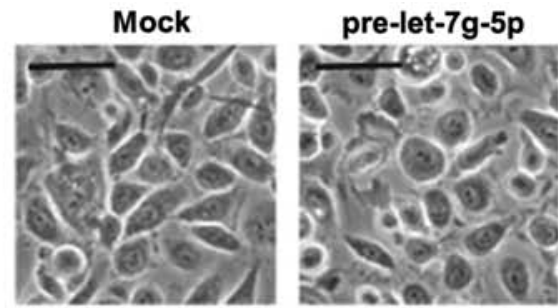

B

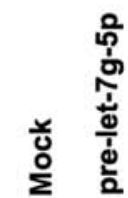

C
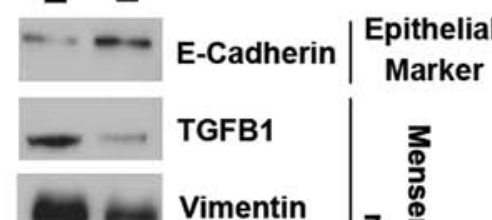

TGFB1

Vimentin

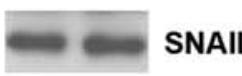

SNAIL

ZEB1

Notch1

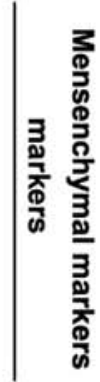

$\beta$-actin

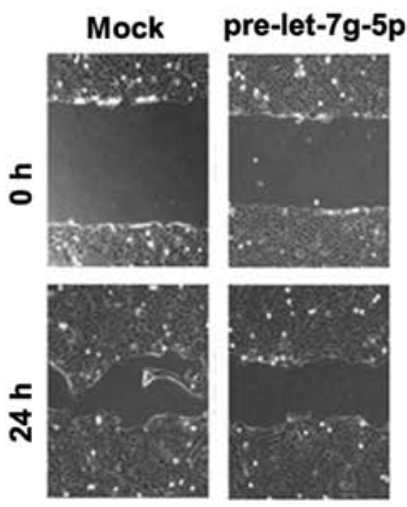

D
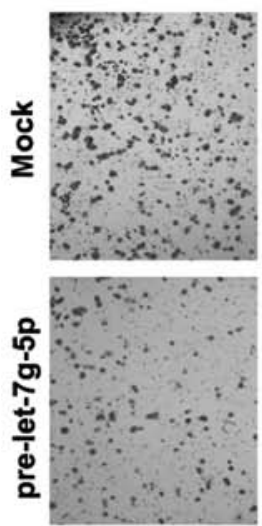

Migration assay
E

Mock

pre-let-7g-5p

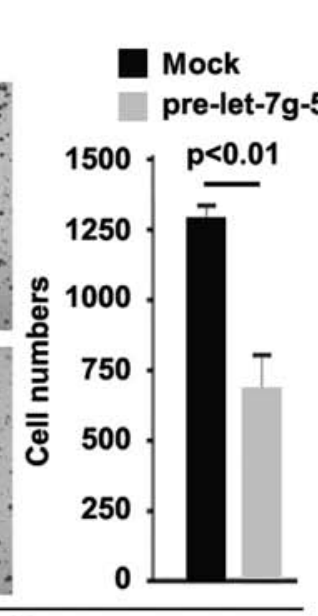
y

Figure 5. Overexpressing let-7g-5p promotes MET in glioblastoma U-87MG cells. (A) U-87MG cells were transfected with pre-let-7g-5p and control miR

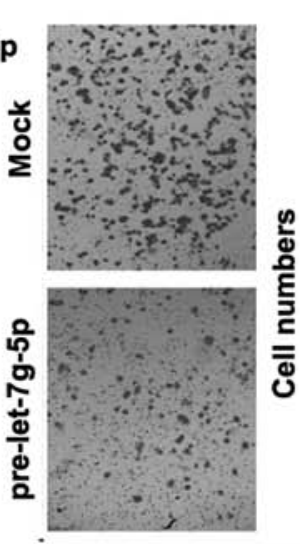

Mock

pre-let-7g-5p

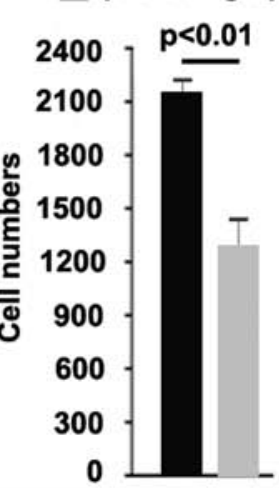

Invasion assay (mock). Cells were then photographed after $72 \mathrm{~h}$ of transfection. $\mathrm{n}=3$. (B) Western blotting for E-cadherin, TGFB1, Vimentin, SNAIL, ZEB1 and Notch1 in U-87MG cells transfected with pre-let-7g-5p and control miR (mock). $\beta$-actin was a loading control. $n=3$. (C) Wound-healing assays for U-87MG cells transfected with pre-let-7g-5p and control miR (mock). The cell layer was photographed. $\mathrm{n}=3$. (D) Migration assays for U-87MG cells transfected with pre-let-7g-5p and control miR (mock). $n=3$. (E) Invasion assays for U-87MG cells transfected with pre-let-7g-5p and control miR (mock). $n=3$.

To identify whether let-7g-5p could inhibit migration and invasion, we performed wound-healing, migration and invasion assays. We found that overexpressing let-7g-5p resulted in decreased migration (Fig. 5C and D) and invasion (Fig. 5E) in the cells.

\section{Discussion}

Recently, it was reported that VSIG4 is highly expressed in glioblastoma and correlated with poor prognosis of high-grade glioma patients (49). However, its role has not been reported in glioblastoma cells. Consistent with the previous report, we found that VSIG4 protein is upregulated in glioblastoma. Ionizing radiation represents the most effective therapy for glioblastoma (50), but radiotherapy remains only palliative (51) because of radio-resistance. Glioma stem cells can promote radio-resistance (18). We showed that overexpressing VSIG4 promoted glioma stem cell phenotypes in U87MG cells, implying that VSIG4 might play an important role in radio-resistance. The emerging role of VSIG4 in glioblastoma response to radiotherapy urges further investigation. Notch1 and Notch 2 can promote radio-resistance of GSCs in glioma 
(52). We found that VSIG4 can evidently promote Notch1 protein expression. The results further indicated that VSIG4 is a potential candidate to prevent radiotherapy resistance. Moreover, VAMP8 can promote temozolomide resistance in human glioma cells (37). Our results also found that VSIG4 can upregulate VAMP8 protein expression in glioblastoma cells, indicating its upregulation might be a cause for temozolomide resistance.

Although the cell origin of cancer stem cells (CSCs) remains to be fully elucidated, mounting evidence has demonstrated that epithelial-to-mesenchymal transition, induced by different factors, is associated with tumor aggressiveness and metastasis and these cells share molecular characteristics with CSCs (53). We found that VSIG4 induced epithelial-to-mesenchymal transition consistent with glioma stem cell phenotypes in glioblastoma cells.

Let-7g-5p is significantly downregulated in the serum of GBM patients and it has been proposed as a tumor suppressive gene in glioblastoma $(11,12)$. Our results showed that its overexpression inhibited VSIG4 protein in glioblastoma cells. Contrary to VSIG4, overexpressing let-7g-5p promoted mesenchymal-epithelial transition and significantly inhibited invasion and migration consistent with the reduction of glioblastoma stem cells phenotypes in U87MG cells.

Elucidating the mechanism that let-7g-5p inhibits epithelial-mesenchymal transition consistent with the reduction of glioma stem cell phenotypes by targeting VSIG4 in glioblastoma will help us to better understand the molecular mechanism of epithelial-mesenchymal transition and glioma stem cells in glioblastoma. Thus, restoration of let-7g-5p may represent a promising therapeutic way to inhibit VSIG4mediated EMT and GSCs regulation. However, the roles of let-7g-5p/VSIG4 need to be further confirmed in vivo.

\section{References}

1. Jemal A, Siegel R, Xu J and Ward E: Cancer statistics, 2010. CA Cancer J Clin 60: 277-300, 2010.

2. Furnari FB, Fenton T, Bachoo RM, Mukasa A, Stommel JM, Stegh A, Hahn WC, Ligon KL, Louis DN, Brennan C, et al: Malignant astrocytic glioma: Genetics, biology, and paths to treatment. Genes Dev 21: 2683-2710, 2007.

3. Stupp R, Hegi ME, Mason WP, van den Bent MJ, Taphoorn MJ, Janzer RC, Ludwin SK, Allgeier A, Fisher B, Belanger K, et al; European Organisation for Research and Treatment of Cancer Brain Tumour and Radiation Oncology Groups; National Cancer Institute of Canada Clinical Trials Group: Effects of radiotherapy with concomitant and adjuvant temozolomide versus radiotherapy alone on survival in glioblastoma in a randomised phase III study: 5-year analysis of the EORTC-NCIC trial. Lancet Oncol 10: 459-466, 2009.

4. Bartel DP: MicroRNAs: Genomics, biogenesis, mechanism, and function. Cell 116: 281-297, 2004.

5. Kim VN: Small RNAs: Classification, biogenesis, and function. Mol Cells 19: 1-15, 2005.

6. Mendell JT: miRiad roles for the miR-17-92 cluster in development and disease. Cell 133: 217-222, 2008.

7. Lu J, Getz G, Miska EA, Alvarez-Saavedra E, Lamb J, Peck D, Sweet-Cordero A, Ebert BL, Mak RH, Ferrando AA, et al: MicroRNA expression profiles classify human cancers. Nature 435: 834-838, 2005.

8. Subramanian S, Lui WO, Lee $\mathrm{CH}$, Espinosa I, Nielsen TO, Heinrich MC, Corless CL, Fire AZ and van de Rijn M: MicroRNA expression signature of human sarcomas. Oncogene 27: 2015-2026, 2008.

9. Sarver AL, Phalak R, Thayanithy V and Subramanian S: S-MED: Sarcoma microRNA expression database. Lab Invest 90: 753-761, 2010 .
10. Ward A, Balwierz A, Zhang JD, Küblbeck M, Pawitan Y, Hielscher T, Wiemann S and Sahin Ö: Re-expression of microRNA-375 reverses both tamoxifen resistance and accompanying EMT-like properties in breast cancer. Oncogene 32: 1173-1182, 2013.

11. Dong L, Li Y, Han C, Wang X, She L and Zhang H: miRNA microarray reveals specific expression in the peripheral blood of glioblastoma patients. Int J Oncol 45: 746-756, 2014.

12. Mao XG, Hütt-Cabezas M, Orr BA, Weingart M, Taylor I, Rajan AK, Odia Y, Kahlert U, Maciaczyk J, Nikkhah G, et al: LIN28A facilitates the transformation of human neural stem cells and promotes glioblastoma tumorigenesis through a proinvasive genetic program. Oncotarget 4: 1050-1064, 2013.

13. Huang Z, Cheng L, Guryanova OA, Wu Q and Bao S: Cancer stem cells in glioblastoma - molecular signaling and therapeutic targeting. Protein Cell 1: 638-655, 2010.

14. Galli R, Binda E, Orfanelli U, Cipelletti B, Gritti A, De Vitis S, Fiocco R, Foroni C, Dimeco F and Vescovi A: Isolation and characterization of tumorigenic, stem-like neural precursors from human glioblastoma. Cancer Res 64: 7011-7021, 2004.

15. Singh SK, Clarke ID, Terasaki M, Bonn VE, Hawkins C, Squire J and Dirks PB: Identification of a cancer stem cell in human brain tumors. Cancer Res 63: 5821-5828, 2003.

16. Singh SK, Hawkins C, Clarke ID, Squire JA, Bayani J, Hide T, Henkelman RM, Cusimano MD and Dirks PB: Identification of human brain tumour initiating cells. Nature 432: 396-401, 2004.

17. Dick JE: Stem cell concepts renew cancer research. Blood 112: 4793-4807, 2008.

18. Bao S, Wu Q, McLendon RE, Hao Y, Shi Q, Hjelmeland AB, Dewhirst MW, Bigner DD and Rich JN: Glioma stem cells promote radioresistance by preferential activation of the DNA damage response. Nature 444: 756-760, 2006.

19. Clement V, Sanchez P, de Tribolet N, Radovanovic I and Ruiz i Altaba A: HEDGEHOG-GLI1 signaling regulates human glioma growth, cancer stem cell self-renewal, and tumorigenicity. Curr Biol 17: 165-172, 2007.

20. Lee J, Son MJ, Woolard K, Donin NM, Li A, Cheng CH, Kotliarova S, Kotliarov Y, Walling J, Ahn S, et al: Epigeneticmediated dysfunction of the bone morphogenetic protein pathway inhibits differentiation of glioblastoma-initiating cells. Cancer Cell 13: 69-80, 2008.

21. Piccirillo SG, Reynolds BA, Zanetti N, Lamorte G, Binda E, Broggi G, Brem H, Olivi A, Dimeco F and Vescovi AL: Bone morphogenetic proteins inhibit the tumorigenic potential of human brain tumour-initiating cells. Nature 444: 761-765, 2006.

22. Nieto MA: The ins and outs of the epithelial to mesenchymal transition in health and disease. Annu Rev Cell Dev Biol 27: 347-376, 2011.

23. Savagner P, Yamada KM and Thiery JP: The zinc-finger protein slug causes desmosome dissociation, an initial and necessary step for growth factor-induced epithelial-mesenchymal transition. J Cell Biol 137: 1403-1419, 1997.

24. Thiery JP: Epithelial-mesenchymal transitions in tumour progression. Nat Rev Cancer 2: 442-454, 2002.

25. Yang $J$ and Weinberg RA: Epithelial-mesenchymal transition: At the crossroads of development and tumor metastasis. Dev Cell 14: 818-829, 2008.

26. Chang CJ, Chao CH, Xia W, Yang JY, Xiong Y, Li CW, Yu WH, Rehman SK, Hsu JL, Lee HH, et al: p53 regulates epithelialmesenchymal transition and stem cell properties through modulating miRNAs. Nat Cell Biol 13: 317-323, 2011.

27. Gravdal K, Halvorsen OJ, Haukaas SA and Akslen LA: A switch from E-cadherin to $\mathrm{N}$-cadherin expression indicates epithelial to mesenchymal transition and is of strong and independent importance for the progress of prostate cancer. Clin Cancer Res 13: 7003-7011, 2007.

28. Hader C, Marlier A and Cantley L: Mesenchymal-epithelial transition in epithelial response to injury: The role of Foxc2. Oncogene 29: 1031-1040, 2010.

29. Lu Y, Chopp M, Zheng X, Katakowski M, Buller B and Jiang F: MiR-145 reduces ADAM17 expression and inhibits in vitro migration and invasion of glioma cells. Oncol Rep 29: 67-72, 2013.

30. Zhang BG, Li JF, Yu BQ, Zhu ZG, Liu BY and Yan M: microRNA-21 promotes tumor proliferation and invasion in gastric cancer by targeting PTEN. Oncol Rep 27: 1019-1026, 2012. 
31. Zhang HY, Li JH, Li G and Wang SR: Activation of ARK5/ miR-1181/HOXA10 axis promotes epithelial-mesenchymal transition in ovarian cancer. Oncol Rep 34: 1193-1202, 2015.

32. Yu J, Ryan DG, Getsios S, Oliveira-Fernandes M, Fatima A and Lavker RM: MicroRNA-184 antagonizes microRNA-205 to maintain SHIP2 levels in epithelia. Proc Natl Acad Sci USA 105: 19300-19305, 2008.

33. Zeppernick F, Ahmadi R, Campos B, Dictus C, Helmke BM, Becker N, Lichter P, Unterberg A, Radlwimmer B and HeroldMende CC: Stem cell marker CD133 affects clinical outcome in glioma patients. Clin Cancer Res 14: 123-129, 2008

34. Suvà ML, Riggi N, Janiszewska M, Radovanovic I, Provero $P$, Stehle JC, Baumer K, Le Bitoux MA, Marino D, Cironi L, et al: $\mathrm{EZH} 2$ is essential for glioblastoma cancer stem cell maintenance. Cancer Res 69: 9211-9218, 2009.

35. Li Y, Li A, Glas M, Lal B, Ying M, Sang Y, Xia S, Trageser D, Guerrero-Cázares H, Eberhart CG, et al: c-Met signaling induces a reprogramming network and supports the glioblastoma stem-like phenotype. Proc Natl Acad Sci USA 108: 9951-9956, 2011.

36. Pietras A, Katz AM, Ekström EJ, Wee B, Halliday JJ, Pitter KL, Werbeck JL, Amankulor NM, Huse JT and Holland EC: Osteopontin-CD44 signaling in the glioma perivascular niche enhances cancer stem cell phenotypes and promotes aggressive tumor growth. Cell Stem Cell 14: 357-369, 2014.

37. Chen Y, Meng D, Wang H, Sun R, Wang D, Wang S, Fan J, Zhao Y, Wang J, Yang S, et al: VAMP8 facilitates cellular proliferation and temozolomide resistance in human glioma cells. Neuro Oncol 17: 407-418, 2015.

38. Gielen PR, Aftab Q, Ma N, Chen VC, Hong X, Lozinsky S, Naus CC and Sin WC: Connexin43 confers Temozolomide resistance in human glioma cells by modulating the mitochondrial apoptosis pathway. Neuropharmacology 75: 539-548, 2013.

39. Sun S, Lee D, Ho AS, Pu JK, Zhang XQ, Lee NP, Day PJ, Lui WM, Fung CF and Leung GK: Inhibition of prolyl 4-hydroxylase, beta polypeptide $(\mathrm{P} 4 \mathrm{HB})$ attenuates temozolomide resistance in malignant glioma via the endoplasmic reticulum stress response (ERSR) pathways. Neuro-oncol 15: 562-577, 2013.

40. Polyak K and Weinberg RA: Transitions between epithelial and mesenchymal states: Acquisition of malignant and stem cell traits. Nat Rev Cancer 9: 265-273, 2009.

41. Zuo JH, Zhu W, Li MY, Li XH, Yi H, Zeng GQ, Wan XX, He QY, Li JH, Qu JQ, et al: Activation of EGFR promotes squamous carcinoma SCC10A cell migration and invasion via inducing EMT-like phenotype change and MMP-9-mediated degradation of E-cadherin. J Cell Biochem 112: 2508-2517, 2011.
42. Jung H, Lee KP, Park SJ, Park JH, Jang YS, Choi SY, Jung JG, Jo K, Park DY, Yoon JH, et al: TMPRSS4 promotes invasion, migration and metastasis of human tumor cells by facilitating an epithelial-mesenchymal transition. Oncogene 27: 2635-2647, 2008.

43. Christiansen JJ and Rajasekaran AK: Reassessing epithelial to mesenchymal transition as a prerequisite for carcinoma invasion and metastasis. Cancer Res 66: 8319-8326, 2006.

44. Lee RC, Feinbaum RL and Ambros V: The C.elegans heterochronic gene lin-4 encodes small RNAs with antisense complementarity to lin-14. Cell 75: 843-854, 1993.

45. Pasquinelli AE, Reinhart BJ, Slack F, Martindale MQ, Kuroda MI, Maller B, Hayward DC, Ball EE, Degnan B, Müller P, et al: Conservation of the sequence and temporal expression of let-7 heterochronic regulatory RNA. Nature 408: 86-89, 2000.

46. Reinhart BJ, Slack FJ, Basson M, Pasquinelli AE, Bettinger JC, Rougvie AE, Horvitz HR and Ruvkun G: The 21-nucleotide let-7 RNA regulates developmental timing in Caenorhabditis elegans. Nature 403: 901-906, 2000

47. Nakajima G, Hayashi K, Xi Y, Kudo K, Uchida K, Takasaki K, Yamamoto $\mathrm{M}$ and $\mathrm{Ju} \mathrm{J}$ : Non-coding microRNAs hsa-let-7g and hsa-miR-181b are associated with chemoresponse to S-1 in colon cancer. Cancer Genomics Proteomics 3: 317-324, 2006.

48. Ji JF, Zhao L, Budhu AL, et al: Let-7g targets collagen type I $\alpha 2$ and inhibits cell migration in hepatocellular carcinoma. Exp Mol Med 43: 298-304, 2011.

49. Xu T, Jiang Y, Yan Y, Wang H, Lu C, Xu H, Li W, Fu D, Lu Y and Chen J: VSIG4 is highly expressed and correlated with poor prognosis of high-grade glioma patients. Am J Transl Res 7: $1172-1180,2015$.

50. Legler JM, Ries LA, Smith MA, Warren JL, Heineman EF, Kaplan RS and Linet MS: Brain and other central nervous system cancers: Recent trends in incidence and mortality. J Natl Cancer Inst 91: 1382-1390, 1999.

51. Garden AS, Maor MH, Yung WK, Bruner JM, Woo SY, Moser RP and Lee YY: Outcome and patterns of failure following limitedvolume irradiation for malignant astrocytomas. Radiother Oncol 20: 99-110, 1991.

52. Wang J, Wakeman TP, Lathia JD, Hjelmeland AB, Wang XF, White RR, Rich JN and Sullenger BA: Notch promotes radioresistance of glioma stem cells. Stem Cells 28: 17-28, 2010

53. Kong D, Li Y, Wang Z and Sarkar FH: Cancer stem cells and epithelial-to-mesenchymal transition (EMT)-phenotypic cells: Are they cousins or twins? Cancers (Basel) 3: 716-729, 2011. 